\title{
IMPACTO DA GOVERNANÇA NA EFICIÊNCIA DA APLICAÇÃO DOS RECURSOS PÚBLICOS COM EDUCAÇÃO
}

\author{
GOVERNANCE IMPACT ON THE EFFICIENCY OF PUBLIC \\ RESOURCE INVESTMENT ON EDUCATION
}

\section{IMPACTO DE LA GOBERNANZA EN LA EFICIENCIA DE LA APLICACIÓN DE LOS RECURSOS PÚBLICOS CON EDUCACIÓN}

\author{
Rodolfo Rocha dos Santos \\ Mestre em Ciências Contábeis - Universidade \\ Federal de Santa Catarina, Florianópolis, Brasil \\ Professor assistente - Universidade Federal Rural \\ do Rio de Janeiro, Seropédica, Brasil \\ Rodolfo.rocha87@hotmail.com \\ Marcelo Machado de Freitas \\ Mestre em Ciências Contábeis - Universidade \\ Federal de Santa Catarina, Florianópolis, Brasil \\ Professor substituto - UFSC, Florianópolis, \\ Brasil \\ mmf.marcelofreitas@gmail.com \\ Ernesto Fernando Rodrigues Vicente \\ Doutor em Ciências Contábeis - Universidade de \\ São Paulo, São Paulo, Brasil \\ Professor associado - Universidade Federal de \\ Santa Catarina, Florianópolis, Brasil \\ ernesto.vicente@ufsc.br
}

Contextus

ISSN 1678-2089

\begin{abstract}
RESUMO
O objetivo desta pesquisa é verificar se as práticas de governança pública têm impacto sobre a eficiência da aplicação dos recursos públicos em educação nos municípios de Santa Catarina. Para isso, aplicou-se um modelo DEA-VRS, tendo como variáveis de input o Gasto per capita com Educação e Cultura dos municípios e o Rendimento Médio Mensal dos Responsáveis pelos Domicílios Particulares Permanentes. As variáveis de output foram a Taxa de Atendimento e a Taxa de Alfabetização de crianças. Em seguida, utilizou-se um modelo de regressão Tobit para verificar o impacto de variáveis de Governança Pública sobre os escores de eficiência encontrados pelo modelo DEA-VRS. Os resultados indicam que a presença dos Conselhos Municipais de Educação e melhores Índices de Responsabilidade Fiscal e Social impactam positivamente a eficiência da aplicação dos recursos públicos em educação. Com isso, percebe-se a importância dessas boas práticas na gestão dos municípios catarinenses.
\end{abstract}

Palavras-chave: governança pública; recursos públicos; eficiência; educação; análise envoltória de dados.

\begin{abstract}
This article analyses the impact of public governance practices on the efficiency of harnessing public resources in Santa Catarina state education. The DEA-VRS model was used having as inputs Education and Culture expenditures per capita and Average Monthly Income of Persons Responsible for Permanent Private Domiciles and as outputs: Enrolment rate and Literacy Rate of Children. In the second step, a Tobit Regression Model was used to find the impact of public governance variables on the efficiency scores found with the DEA-VRS model. It has been found that the presence of Municipal Councils of Education and better Fiscal and Social Responsibility Indices impact positively the efficiency in using public resources for education. Thus, it seems important for the good management of the cities of Santa Catarina to implement these public governance practices.
\end{abstract}


Keywords: public governance; public resources; efficiency; education; data envelopment analysis.

\section{RESUMEN}

El objetivo de esta investigación es verificar si las prácticas de gobierno público tienen impacto sobre la eficiencia de la aplicación de los recursos públicos en educación en los municipios de Santa Catarina. Para ello, se aplicó un modelo DEA-VRS, teniendo como variables de input el Gasto per cápita con Educación y Cultura de los municipios y el Rendimiento Medio Mensual de los Responsables de los Domicilios Particulares Permanentes. Las variables de output fueron la Tasa de Atención y la Tasa de Alfabetización de niños. A continuación, se utilizó un modelo de regresión Tobit para verificar el impacto de variables de Gobernanza Pública sobre los escores de eficiencia encontrados por el modelo DEA-VRS. Los resultados indican que la presencia de los Consejos Municipales de Educación y mejores Índices de Responsabilidad Fiscal y Social impactan positivamente la eficiencia de la aplicación de los recursos públicos en educación. Con ello, se percibe la importancia de esas buenas prácticas en la gestión de los municipios catarinenses.

Palabras claves: gobernanza pública; recursos públicos; eficiencia; educación; análisis envoltorio de datos.

\section{INTRODUÇÃO}

Inúmeros fenômenos foram responsáveis pelas transformações ocorridas no mundo atual, incluindo a recente crise econômica e financeira, o que acaba por exigir que o Estado se reinvente em um novo modelo para melhorar seu desempenho (MATIAS-PEREIRA, 2010). O autor destaca a crise do Welfare State nos países desenvolvidos, a crise do desenvolvimentismo nos países “periféricos", o esgotamento do modelo econômico, a fragilidade do modelo político, e a deficiência do modelo administrativo como catalisadores dessa mudança.

Nesse novo cenário construído ao longo dos últimos trinta anos, o Estado passa a reafirmar seu papel de apoio ao mercado, por medir, regular, estimular e promover intervenções e regulações, o que exige dos governantes e administradores públicos a adoção de ações inovadoras na gestão do setor público (MATIAS-PEREIRA, 2010). A relação entre Estado e sociedade passa a ser vista por outra perspectiva, com aquele deixando de desempenhar o papel somente de governo e adotando um papel de governança (KISSLER; HEIDEMANN, 2006).

Governança pública se apoia em duas perspectivas distintas: uma associada ao movimento do New Public Management (NPM) (a adoção de práticas da iniciativa privada) e com um maior enfoque em resultados, sem garantir que estes sejam alcançados por meio de processos mais democráticos. A governança pública neodesenvolvimentista busca a melhoria da democracia e se orienta para as formas de interação entre o Estado e a sociedade, envolvendo atores sociais e oferecendo maior destaque às organizações da sociedade civil, na busca pelo desenvolvimento em conjunto e não somente pela perspectiva econômica (MATIASPEREIRA, 2010; DIAS; CARIO, 2014).

Kormendi e Mequire (1985) foram os primeiros a relacionarem práticas de governança pública com crescimento econômico de uma nação. Os autores introduziram uma medida de 
liberdade civil em seu conjunto de variáveis e observaram uma correlação positiva com o crescimento econômico. Desde então, outros estudos surgiram nesse cenário, como: Mauro (1995), para quem a corrupção reduz o nível de investimentos de um país; Knack e Keefer (1995), que constataram terem as práticas de governança maior impacto sobre o crescimento de uma nação do que sobre a acumulação de capital; e Olson, Sarna e Swamy (2000), que mostraram a ligação negativa entre tais práticas e o crescimento mais lento da produtividade.

Rodríguez-Pose e Garcilazo (2015) fizeram uso de modelos econométricos para medir a influência da qualidade da governança no crescimento econômico de 169 regiões da UE. Afonso e Venancio (2016) verificaram a relevância da formação das zonas pendulares na eficiência das despesas públicas regionais nos países da UE. Araújo Júnior et al. (2017) mensuraram o nível de ineficiência das escolas públicas dos estados do Nordeste e identificaram seus possíveis determinantes.

Aos poucos, a literatura foi percebendo que, por trás da diferença do crescimento econômico entre os países, existia algo além da acumulação de capital, como os modelos clássicos e neoclássicos sugerem (MEON; WEILL, 2005). Nesse sentido, Meon e Weill (2005) conseguem relacionar a existência de melhores índices de governança com a melhor eficiência do Estado. No entanto, percebe-se um entendimento mais macroeconômico acerca desse fenômeno, o que desperta o interesse de entender se ele também é percebido no nível microeconômico.

De acordo com Costa et al. (2015), o fator econômico não deve ser o único a ser levado em conta pelo Estado para o desenvolvimento de determinada região, o qual deve ser multidimensional e socioeconômico. Os mesmos autores apontam como dever do Estado proporcionar um ambiente onde as pessoas possam usufruir de vidas longas, saudáveis e criativas. Nessa perspectiva, o desenvolvimento deveria visar a melhorias do bem-estar e da qualidade de vida, principalmente oferecendo condições ideais de saúde e educação (DIAS; CARIO, 2014; COSTA et al., 2015).

Dentro desse escopo, a educação, nesta pesquisa, se apresenta como preocupação microeconômica, que apresenta diversos entraves para se desenvolver. Um dos problemas comumente debatidos pela academia é a falta de eficiência ao utilizar os recursos disponíveis. Dentre as diversas metodologias a permitir avaliar a eficiência, Peña (2008) evidencia que o método de Análise Envoltória de Dados (Data Envelopment Analysis - DEA) tem sido aplicado com sucesso em estudos da administração pública, pois permite comparar os insumos (inputs) 
e os produtos (outputs) de cada unidade e determina os índices de eficiência relativa de cada unidade analisada. De acordo com Curi et al. (2014) esses índices possibilitam delinear as melhores práticas, as unidades ineficientes e as mudanças necessárias no que diz respeito aos níveis de insumos e produtos para que as unidades consideradas ineficientes se tornem eficientes, podendo ser utilizado para identificar recursos ociosos ou inutilizados e para formular políticas de redução de custos.

O problema que este trabalho se propõe investigar gira em torno do fato de que a não adoção de medidas de governança por parte das instituições públicas e do governo pode prejudicar a eficiência na aplicação dos recursos públicos, o que ocasiona menor benefício à sociedade e menor crescimento da economia. Diante disso, o desconhecimento da influência das práticas de governança pública na gestão municipal pode reduzir o bem-estar social proporcionado pelas funções do Estado. Para nortear a análise deste problema, formula-se a seguinte questão de pesquisa: práticas de governança pública afetam a eficiência da aplicação dos recursos públicos com educação nos municípios de Santa Catarina? Dessa maneira, este estudo objetiva identificar se a adoção de práticas de governança pública melhora a eficiência da aplicação dos recursos públicos na educação básica.

\section{REFERENCIAL TEÓRICO}

\subsection{Governança pública e educação}

As discussões em torno da governança pública ainda levantam diversas divergências quanto a sua conceitualização e concepção. Nos tempos modernos, o conceito de governança tornou-se conceito multidimensional, para o qual não é fácil encontrar uma definição comum e amplamente aceita. O termo se expandiu e hoje em dia é encontrado em diferentes áreas, tais como com referência a empresas privadas (governança corporativa), administrações públicas (governança pública), bem como instituições internacionais e suas políticas e recomendações aos países membros (governança global) (CEPIKU, 2008). Para Cepiku (2008), o conceito de governança vem sendo tão utilizado que alguns autores chegam a mencionar uma indústria do termo, embora ainda seja grande a dificuldade para encontrar um acordo.

Tem-se, por um lado, o New Public Management (NPM), cujo objetivo principal é a adequação e a transparência dos alicerces gerenciais desenvolvidos no setor corporativo para o público (MATIAS-PEREIRA, 2010). Rhodes (1996) ainda complementa que o NPM prega a 
competição de mercado como estrutura de incentivo para a prestação de serviços públicos. O NPM visa incentivar a redução do tamanho da máquina administrativa, aumentar sua eficiência e criar mecanismos que busquem delegar responsabilidades aos atores políticos (MATIASPEREIRA, 2010). Dessa maneira, com a implementação do NPM, diminui-se a burocracia, aumentam-se a livre concorrência e a gestão participativa, bem como se melhora a eficiência do Estado (RHODES, 1996).

Por outro lado, para Kissler e Heidemann (2006), o conceito original de governança pública está mais atrelado ao entendimento associado ao debate político desenvolvimentista, sendo o termo utilizado para designar políticas de desenvolvimento guiadas por conjecturas sobre elementos estruturais como gestão, responsabilidades, transparência e legalidade do setor público. Tais elementos são considerados essenciais para o desenvolvimento de todas as sociedades. Ronconi (2011) corrobora essa visão, ao afirmar que governança pública é um projeto político democratizante no qual se enxerga a participação da sociedade civil como indispensável para consolidar a democracia, participação essa resultante da conquista de segmentos sociais que buscam por novas formas de participação e exercício da cidadania.

Para Lynn Junior e Malinowska (2018) governança pública está relacionado a redes de autores autônomos, mas qualificados para atividades coletivas, ocorrendo além dos limites da influência direta do governo. Já para Oliveira e Pisa (2015), a governança pública deve apresentar duas características em comum com a governança corporativa: a evidenciação dos resultados e a presença de auditoria externa, práticas importantes para a boa governança das empresas no setor privado.

Por fim, Ronconi (2011) aponta uma confluência entre os dois jeitos de pensar governança pública no que diz respeito à construção de cidadania e participação da sociedade civil ativa e propositiva, por mais que apontem para direções opostas e até mesmo antagônicas. Também pode-se perceber um discurso acerca da melhoria da eficiência da máquina pública por meio da governança pública, tanto na visão do NPM, quanto na neodesenvolvementista. É nesse ponto comum que o trabalho se propõe operar, sem se preocupar em definir governança pública por uma das duas visões.

Considerando a educação como direito de todos e dever do Estado garantido pela Constituição Federal de 1988, acredita-se que se devam utilizar práticas capazes de aumentar a eficiência ao aplicar recursos voltados a essa função estatal, especificamente com práticas de governança pública. Para Lopes e Toyoshima (2008), uma maior alocação dos recursos 
públicos na educação, saneamento básico, saúde e habitação é fundamental para melhorar o desenvolvimento e reduzir as disparidades. De acordo com Whiteley (2000), altos níveis de educação melhoram o nível de capital humano de um país gerando crescimento econômico no longo prazo.

Porém, somente conhecer a aplicação dos recursos públicos com provisões de bens e serviços não é suficiente, sendo importante saber o resultado gerado por essa aplicação e se esse resultado poderia ser alcançado com menores custos para o contribuinte (REZENDE; CUNHA; BEVILACQUA, 2010). Ainda nesse sentido, Will (2014) afirma que nem sempre o maior dispêndio de recursos resulta em uma maior qualidade de suas aplicações, daí a importância de estudar os resultados da aplicação dos recursos públicos. O autor complementa que esses resultados podem ser medidos pela eficiência na aplicação por parte dos agentes públicos.

Para Peña (2008), eficiência é a combinação ótima dos insumos e métodos necessários (inputs) ao processo produtivo que resulte no máximo de produtos possíveis (outputs), isto é, eficiência é a capacidade de fazer certo as coisas, de minimizar a relação insumo e produto e, desse modo, otimizar a utilização de recursos. Mello et al. (2005) afirmam que eficiência é comparar o que foi produzido, dados os recursos disponíveis, com o que poderia ter sido produzido com os mesmos recursos.

Independentemente da visão de governança pública tida pela teoria, esse trabalho se prendeu no que diz respeito à melhoria da eficiência dos gastos públicos por conta de melhores práticas de governança pela administração pública.

\subsection{Estudos anteriores}

Diversos trabalhos já foram feitos com o intuito de medir a eficiência da aplicação dos recursos destinados à educação. Várias metodologias foram aplicadas para tanto, dentre elas a DEA. Charnes, Cooper e Rhodes (1978) desenvolveram a DEA com base no trabalho de Farrel (1957), e desde então inúmeros trabalhos se utilizaram dessa ferramenta de maneira satisfatória para medir a eficiência pública (PEÑA, 2008).

Contudo, poucos estudos têm investigado a relação entre governança pública e eficiência dos gastos públicos com educação. No entanto, alguns deles já observaram uma relação existente entre governança pública e a eficiência em outras áreas, como é o caso de Asatryan e Witte (2015). Os autores estudaram o papel da democracia direta e participativa na 
provisão eficiente de bens públicos por parte dos municípios do estado alemão da Bavária. Para tanto, o estudo aplicou um modelo de Free Disposal Hull (FDH) para medir a eficiência dos municípios e posteriormente verificar se municípios que apresentaram um maior nível de participação da sociedade civil nas suas tomadas de decisão atingiram maiores escores de eficiência. Como resultado, os autores constataram que práticas democráticas mais diretas estão associadas com uma maior eficiência do governo na provisão dos bens e serviços, o que sugere que práticas de governança pública surte efeitos positivos na eficiência do Estado.

Em uma visão macroeconômica, Meon e Weill (2005) testaram a relação entre governança e eficiência técnica em uma amostra de 62 países desenvolvidos e em desenvolvimento. Os autores calcularam a eficiência dos países com uma abordagem de fronteira estocástica e testaram o impacto de seis indicadores de governança sobre a escala calculada por eles. Em seus resultados, os autores mostram que uma melhor governança está associada a uma melhor eficiência desenvolvida pelo Estado.

Chang (2014) verificou a relação da governança pública com a eficiência de empresas geradoras de energia elétrica da China e de Taiwan. $\mathrm{O}$ autor construiu um modelo DEA para medir a eficiência de produção, eficiência ambiental e eficiência geral das empresas de energia elétrica dos referidos países e, através do modelo de regressão Tobit, examinaram o impacto das variáveis "controle da corrupção" e "eficácia governamental" das escalas de eficiência gerada pelo modelo DEA proposto. Os dados demonstraram que a boa qualidade da governança pública impacta a melhoria da eficiência da indústria de energia elétrica chinesa.

No cenário nacional, Silva e Almeida (2012) fizeram uso da DEA para construir uma escala de eficiência dos municípios do Rio Grande do Norte quanto aos gastos públicos com educação. Por meio do modelo de regressão Tobit, verificaram o impacto de diversas variáveis na ineficiência atingida pelas DMUs, dentre elas a presença de Conselhos Municipais de Educação. Os autores observaram uma relação negativa da ineficiência dos municípios com a variável em questão, o que sugere o incremento da eficiência da máquina pública com base em práticas de governança.

A Tabela 1 apresenta alguns trabalhos que utilizaram da metodologia DEA para medir a eficiência da aplicação dos recursos públicos com educação no cenário brasileiro. 
Tabela 1 - Relação de estudos nacionais que abordaram a eficiência dos gastos com educação \begin{tabular}{l|l}
\hline Autor, ano, revista ou anais & Objetivos, inputs, outputs e metodologia \\
\hline
\end{tabular}

\begin{tabular}{|c|c|}
\hline $\begin{array}{l}\text { (FARIA; JANUZZI; SILVA, } \\
\text { 2008) } \\
\text { RAP-Revista de Administração } \\
\text { Pública }\end{array}$ & $\begin{array}{l}\text { Buscaram, utilizando a DEA, analisar a relação existente entre as despesas } \\
\text { sociais realizadas nos municípios do Rio de Janeiro (gastos públicos com } \\
\text { educação e cultura, saúde e saneamento) e indicadores da condição de vida } \\
\text { da população ali residente. O trabalho criou uma série de modelos até } \\
\text { selecionar dois com maior poder de discriminação. As variáveis utilizadas } \\
\text { como inputs e outputs para calcular a eficiência da educação foram: - input: } \\
\text { gastos per capita com educação e cultura e rendimento médio mensal dos } \\
\text { responsáveis pelos domicílios particulares permanentes; - outputs: taxa de } \\
\text { alfabetização de } 10 \text { a } 14 \text { anos; proporção de crianças de } 2 \text { a } 5 \text { anos } \\
\text { matriculadas em creches ou escolas de educação infantil. }\end{array}$ \\
\hline $\begin{array}{l}\text { (LOPES; TOYOSHIMA, 2008) } \\
\text { Anais de seminário sobre a } \\
\text { economia mineira }\end{array}$ & $\begin{array}{l}\text { Os autores calcularam a eficiência técnica da gestão dos recursos com saúde } \\
\text { e educação no estado de Minas Gerais utilizando a metodologia DEA e } \\
\text { verificaram os impactos e determinantes dessa eficiência. Constataram que a } \\
\text { eficiência da aplicação dos recursos públicos tende a elevar o bem-estar e } \\
\text { reduzir a desigualdade do estado. Para o cálculo da eficiência com educação } \\
\text { dos municípios mineiros, os autores utilizaram como inputs gastos per capita } \\
\text { com educação e cultura e renda média mensal dos chefes de famílias e como } \\
\text { outputs percentual de docentes com curso superior no ensino fundamental e } \\
\text { taxa de alfabetização. }\end{array}$ \\
\hline $\begin{array}{l}\text { (WILBERT; D'ABREU, 2013) } \\
\text { ASAA - Advances in Scientific } \\
\text { and Applied Accounting }\end{array}$ & $\begin{array}{l}\text { Wilbert e D’Abreu (2013) aplicaram a DEA para medir a eficiência dos } \\
\text { gastos públicos com educação fundamental dos municípios do estado de } \\
\text { Alagoas no período de } 2007 \text { a } 2011 \text {. Evidenciaram nos resultados de sua } \\
\text { pesquisa que os municípios que compuseram a fronteira de eficiência foram } \\
\text { aqueles com as piores condições em termos de riqueza média e nível } \\
\text { educacional, enquanto os menos eficientes foram aqueles com a melhor } \\
\text { condição em termos de PIB per capita e elevados gastos por aluno, porém } \\
\text { apresentaram os piores desempenhos no Ideb (Índice de Desenvolvimento da } \\
\text { Educação Básica) de } 2011 \text {. } \\
\text { Input: gastos públicos com educação; output: nota dos municípios alagoanos } \\
\text { no Ideb de } 2011 \text { para a } 4^{\mathrm{a}} \text { e } 8^{\mathrm{a}} \text { série do ensino fundamental em escolas } \\
\text { municipais. }\end{array}$ \\
\hline
\end{tabular}

Buscaram avaliar a eficiência técnica dos gastos públicos dos gastos municipais em educação, saúde e assistência social para os municípios cearenses referentes aos anos de 2005 utilizando a metodologia DEA. Os (MACHADO JUNIOR; IRFFI; autores encontraram que, se analisarem a eficiência relativa pelo conjunto de BENEGAS, 2011) Planejamento e Políticas todas as áreas, os municípios cearenses apresentam um bom desempenho; já

Públicas
analisando suas eficiências por área, os municípios apresentam uma baixa eficiência técnica. Os autores utilizaram as seguintes variáveis para o cálculo da eficiência com educação: - input: gasto per capita com educação e cultura; - outputs: número de estabelecimentos de educação infantil, taxa de alfabetização de educação infantil e taxa de escolarização.

Silva et al. (2012) analisaram a eficiência de alocação dos recursos destinados à educação, saúde e habitação nos municípios de Minas Gerais, também aplicando a metodologia DEA. Concluíram que as práticas de gestão nos

(SILVA et al., 2012) Contabilidade, Gestão municípios mineiros devem ser revisadas por conta de os métodos adotados Governança

$e$ apresentarem uma baixa média dos escores de eficiência.

Inputs: gasto per capita com educação e cultura e Produto Interno Bruto (PIB) per capita; outputs: taxa de atendimento das crianças de 4 a 6 anos; taxa de crescimento das crianças de 7 a 14 anos e taxa de atendimento de adolescentes de 15 a 17 anos.

Fonte: elaboração própria. 


\section{PROCEDIMENTOS METODOLÓGICOS}

A metodologia da pesquisa pode ser dividida em duas etapas distintas: na primeira, utiliza-se a DEA para construir os índices de eficiência dos gastos públicos com educação nos municípios do estado de Santa Catarina; na segunda, utilizam-se os indicadores de eficiência como variável dependente em um modelo de regressão Tobit, com o intuito de avaliar se variáveis de governança constroem uma relação de significância com tais indicadores.

\subsection{Análise envoltória de dados}

Conforme Lins e Meza (2000), a metodologia DEA foi proposta por Charnes, Cooper e Rhodes (1978) a partir do trabalho de Farrell (1957). A DEA foi proposta para avaliar a eficiência inerente de unidades tomadoras de decisões homogêneas (DMUs - Decision Making Units) tendo como referencial a transformação de inputs em outputs (CHARNES; COOPER; RHODES, 1978).

A DEA fornece um resultado de eficiência relativa dentro de 0 a 1 para cada uma das DMUs, apontando aquelas que apresentam escore igual a 1 como os benchmarkings e aquelas com eficiência inferior como relativamente ineficientes. A fronteira de eficiência é composta pelas DMUs eficientes, e é a partir dela que se definem metas de desempenho para as DMUs ineficientes (MACEDO; CASA NOVA; ALMEIDA, 2009).

A vantagem de utilizar a DEA é que ela permite avaliar o desempenho de organizações que operam em mercados cujos preços não estão disponíveis (ZHU, 2000). Nestes casos, os indicadores de desempenho habituais, como a rentabilidade e as taxas de retorno, não podem ser usados para medir o desempenho econômico de uma instituição com precisão (ABBOTT, DOUCOLIAGOS, 2003). Will (2014) ainda destaca que a sua utilização se adequa à utilização de insumos ou à geração de produtos, com seus valores não podendo ser mensurados em termos monetários. Por conta disso, a DEA tem sido aplicada com expressivo sucesso em estudos sobre eficiência na Administração Pública (PEÑA, 2008).

Há duas abordagens clássicas do modelo DEA: o CCR (Charnes, Cooper e Rhodes), também chamado de CRS (Constant Returns to Scale), que aceita como hipótese um retorno constante de escala (CHARNES; COOPER; RHODES, 1978); e o BCC (Banker, Charnes e Cooper), conhecido como VRS (Variable Returns to Scale), que considera situações de 
eficiência na produção com variação de escala e não assume proporcionalidade entre inputs e outputs (BANKER; CHARNES; COOPER, 1984).

No modelo CCR, qualquer variação nos inputs acarreta uma variação proporcional dos outputs, suposição esta denominada axioma da proporcionalidade (TSCHAFFON; MEZA, 2014). Já o modelo BCC pressupõe que as DMUs assumam retornos variáveis de escala, formando uma fronteira convexa, sem proporcionalidade entre inputs e outputs (MELLO et al., 2008; TSCHAFFON; MEZA, 2014). Esse modelo permite a projeção de cada DMU ineficiente sobre a fronteira de eficiência (envoltória) estabelecida conforme os diferentes portes de DMUs, onde a produtividade máxima é estabelecida conforme as escalas de produção. O BCC surgiu na divisão da eficiência do modelo CCR em duas componentes: a eficiência técnica e a eficiência de escala (BELLONI, 2000; MACEDO; CASA NOVA; ALMEIDA, 2009; WILL, 2014). Além das abordagens clássicas mencionadas anteriormente, o DEA admite orientações quanto aos inputs e outputs. Na orientação input se verifica o quanto se conseguiria reduzir o número de recursos para se produzir o mesmo nível de produtos, já na orientação output se verifica o quanto se pode maximizar o nível de produção se mantendo o mesmo nível de insumos (TSCHAFFON; MEZA, 2014).

A escolha das variáveis para o cálculo da eficiência relativa se deu com base na função alocativa do Estado orientando-se nas necessidades de atendimento aos cidadãos no âmbito da educação, e com base na análise criteriosa de diferentes referencias teóricos que já abordaram o assunto. Tomou-se como referência alguns quesitos já validados por Lopes e Toyoshima (2008), Faria, Januzzi e Silva (2008), Machado Junior, Irffi e Benegas (2011), Silva et al. (2012) e Silva e Almeida (2012).

As variáveis foram divididas em inputs e outputs apresentados na Tabela 2. Como inputs selecionou-se Gasto per capita com educação e cultura (GpcEdu\&C) dos municípios e rendimento médio mensal dos responsáveis pelos domicílios particulares permanentes (Renda) de cada município.

Como outputs selecionaram-se: a taxa de atendimento às crianças de 0 a 5 anos (TaxAt0$5 \%$ ) de cada município, a taxa de atendimento às crianças de 5 a 6 anos (TaxAt5-6\%), a taxa de atendimento às crianças de 7 a 14 anos (TaxAt7-14\%) e a taxa de alfabetização de crianças de 11 a 14 anos (TaxAlf11-14\%).

A escolha das variáveis TaxAt0-5\%, TaxAt5-6\% e TaxAt7-14\% deu-se por conta da responsabilidade delegada aos municípios pela Constituição Brasileira de 1988 em seu art. 211, 
$\S 2^{\circ}$ quanto à educação fundamental e à infantil. Escolheu-se a taxa de alfabetização de crianças de 11 a 14 anos como variável de output por ela estar diretamente ligada ao fato de a educação voltada para tal faixa etária ser de responsabilidade dos municípios, por mais que ainda exista uma participação de verbas estaduais (FARIA; JANNUZZI; SILVA, 2008).

Tabela 2 - Variáveis

\begin{tabular}{l|l}
\hline Inputs & Autores que as utilizaram \\
\hline \hline GpcEdu\&C & $\begin{array}{l}\text { Faria, Januzzi e Silva (2008); Lopes e Toyoshima, (2008); Machado Junior, Irffi e } \\
\text { Benegas, (2011) e Silva et al. (2012) }\end{array}$ \\
\hline Renda & Faria, Januzzi e Silva (2008); Lopes e Toyoshima (2008) \\
\hline Outputs & Autores que as utilizaram \\
\hline \hline TaxAt0-5\% & Faria, Januzzi e Silva (2008); Silva et al. (2012) \\
\hline TaxAt5-6\% & Silva et al. (2012) \\
\hline TaxAt7-14\% & Silva et al. (2012) \\
\hline TaxAlf11-14 anos\% & Faria, Januzzi, Silva (2008); Machado Junior, Irffi, Benegas (2011) \\
\hline
\end{tabular}
Fonte: elaboração própria.

Optou-se por utilizar a orientação output, que mede o quanto a produção pode ser expandida sem alterar os inputs (no caso, o quanto as taxas de atendimento e alfabetização ainda podem ser ampliadas para os municípios ineficientes). Optou-se também pela aplicação do BCC ou VRS, com base na recomendação de Souza Júnior e Gasparini (2006), com o intuito de considerar a disparidade de tamanho existente entre os municípios catarinenses.

O cálculo dos escores de eficiência foram feitos com o software Open Source DEA (OSDEA) versão 0.2.

\subsection{Impacto da governança pública}

Segundo Hoff (2007), tanto o modelo de regressão Tobit, quanto o modelo de Mínimos Quadrados Ordinários (MQO) são suficientes para o chamado "segundo estágio" da Análise Envoltória de Dados. Porém, Sueyoshi, Goto e Omi (2010) afirmam que o Tobit é mais adequado para a segunda fase da DEA, pois o MQO acaba se tornando tendencioso pela característica truncada da eficiência. Portanto, para esse trabalho optou-se por utilizar o modelo de regressão Tobit, apresentado na equação a seguir, juntamente com as variáveis independentes.

$$
D E A=\alpha+\beta_{1} X_{1}+\beta_{2} X_{2}+\beta_{3} X_{3}+\varepsilon
$$

Onde: 
$\alpha$ é a constante da função;

$\beta_{1}, \beta_{2}$ e $\beta_{3}$ são os coeficientes estimados da regressão;

$\varepsilon$ é o fator de erro da regressão; e

$\mathrm{X}_{1}, \mathrm{X}_{2}$ e $\mathrm{X}_{3}$ são as variáveis independentes do modelo, definidas a seguir: $\mathrm{X}_{1}$ - Presença de Conselho Municipal de Educação (PCME); $\mathrm{X}_{2}$ - Índice de Responsabilidade Fiscal, Social e de Gestão educacional dos municípios (IRFS); $X_{3}$ - Presença de Conselho Municipal de acompanhamento e controle social do FUNDEB.

A variável Presença de Conselho Municipal de Educação (CME) é uma variável dummy utilizada como proxy para identificação da prática de governança participativa na gestão da educação municipal. Teixeira (2004) discute que os CMEs constituem o espaço próprio de deliberações cuidadosas, que refletem de alguma forma as exigências da sociedade. Para Silva e Almeida (2012), os CME, por terem participação da comunidade na gestão do ensino, podem estabelecer metas e diretrizes para a política educacional do município. Por tanto, espera-se que a presença de CME resulte em um maior controle e fiscalização da aplicação dos recursos públicos com educação, provocando efeitos positivos na eficiência dos gastos municipais nessa função. Em geral, é esperado que a introdução de elementos democráticos diretos para complementar as instituições da democracia representativa aumente a eficiência do governo (ASATRYAN, WITTE, 2015). Silva e Almeida (2012) também fizeram uso dessa variável em seu trabalho para medir a influência dela na escala dos municípios estudados.

O Índice de Responsabilidade Fiscal, Social e de Gestão tem como objetivo compreender e estimular a melhoria das gestões municipais (CRUZ et al., 2012). O IRFS reflete o desempenho anual dos municípios sob três óticas: fiscal, social e gestão. Seu objetivo consiste em disseminar a cultura da responsabilidade fiscal associada à responsabilidade social, de modo que municípios apresentando melhores práticas de responsabilidade fiscal e social alcançam maiores IRFS (CNM, 2015). Esse trabalho se utilizou do IRFS Social, que por sua vez é composto pelo IRFS da educação e o IRFS da saúde. Por não se ter acesso ao IRFS da educação separadamente, se optou por utilizar o IRFS Social como um todo. Espera-se que municípios que apresentam maiores IRFS Social tenham melhores práticas de gestão e consequentemente obtenham maiores índices de eficiência na aplicação dos seus recursos com educação. Cruz et al. (2012) utilizaram essa variável como explicativa para o nível de transparência dos municípios quanto suas e-informações. 
O Fundo de Manutenção e Desenvolvimento da Educação Básica (FUNDEB) é um fundo especial e de natureza contábil criado pela Emenda Constitucional nº 53/2006 e regulamentado pela Lei $\mathrm{n}^{\mathrm{o}} 11.494 / 2007$, formado por parcela financeira de recursos federais e por recursos provenientes dos impostos e das transferências dos Estados, Distrito Federal e Municípios e todo o recurso gerado é redistribuído para ser aplicado exclusivamente na educação básica (FNDE, 2015). A lei prevê a possibilidade de criação de Conselhos Municipal de acompanhamento e controle social do FUNDEB e esse trabalho utilizou-se como variável a presença de tais conselhos como uma prática de governança pública. Para tanto, optou-se pelo uso de uma dummy e municípios que apresentaram registro regularizado no ano de 2010 junto ao sistema CACS-FUNDEB foi atribuído valor 1 e para os que não apresentaram tal situação foi atribuído valor 0. Espera-se uma relação positiva da escala de eficiência construída pelo presente trabalho e a presença da variável descrita acima.

A principal limitação do estudo relaciona-se com a construção do modelo de eficiência quanto à seleção das variáveis de inputs e outputs que o compuseram. Essa seleção fica passível de discussão, embora se tenham utilizado critérios já validados por outros autores. Por mais que se acredite que tais variáveis reflitam de maneira satisfatória os resultados que cabem à responsabilidade municipal quanto à educação, correu-se o risco de deixar de fora outras informações que também podem ser relevantes. Outra limitação quanto ao modelo é o uso de todas as variáveis de inputs e outputs. De acordo com Lins e Meza (2000), a introdução de um grande número de variáveis resulta em uma maior explicação das diferenças das DMUs, porém fará com que um número maior de DMUs esteja na fronteira de eficiência, tendo assim pouco poder de discriminação. Entretanto, para esse trabalho se optou por utilizar o modelo construído com todas as variáveis selecionadas como inputs e outputs.

\subsection{Universo, amostra e base de dados}

A amostra do trabalho foi composta por 277 municípios dos 295 do estado de Santa Catarina, pelo fato de não ter sido encontrado um ou mais dados para os municípios de Arroio Trinta, Balneário Piçarras, Balneário Rincão, Bandeirante, Bom Jesus do Oeste, Herval d Oeste, Iomerê, Ipira, Lajeado Grande, Ouro, Peritiba, Pescaria Brava, Presidente Castello Branco, São Bernardino, São Miguel da Boa Vista, Serra Alta, Tigrinhos e Treviso, porém a amostra compreende $93,90 \%$ de todos os municípios e mais de $98 \%$ da população do estado. 
As variáveis socioeconômicas e estatísticas da educação foram extraídas do Atlas do Desenvolvimento Humano, que por sua vez se utiliza de dados secundários do Instituto Brasileiro de Geografia (IBGE), Instituto de Pesquisa Econômica Aplicada (IPEA), Instituto Nacional de Estudos e Pesquisas Educacionais Anísio Teixeira (INEP). Os dados referentes aos gastos públicos com educação de cada município foram obtidos junto à Secretaria do Tesouro Nacional (STN). A variável PCME foi obtida junto ao site da União Nacional dos Conselhos Municipais de Educação (UNCME), e a variável IRFS Social foi obtida junto à Confederação Nacional de Municípios (CNM). Os dados correspondem ao ano de 2010, último censo, por conta da falta de disponibilidade de informações mais atuais dos municípios catarinenses para todas as variáveis selecionadas.

\section{RESULTADOS}

\subsection{Análise descritiva}

A análise das estatísticas descritivas da escala de eficiência gerada pelo modelo DEA foi feita por mesorregião do estado catarinense e se apresenta na Tabela 3. O escore médio obtido por Santa Catarina foi de 0,9945 com desvio-padrão de 0,0054, com os escores variando de 0,9667 até 1 . Dentre os 277 municípios catarinenses que compuseram a amostra, 74 ficaram na fronteira de eficiência atingindo o índice igual a 1 e foram considerados benchmarks para o restante dos municípios que ficaram ineficientes.

Os municípios que compuseram a escala de eficiência foram: Abdon Batista; Águas Frias; Alto Bela Vista; Anchieta; Anitápolis; Arabutã; Bela Vista do Toldo; Belmonte; Benedito Novo; Bombinhas; Braço do Trombudo; Caibi; Calmon; Celso Ramos; Chapadão do Lageado; Cordilheira Alta; Cunhataí; Dona Emma; Doutor Pedrinho; Flor do Sertão; Frei Rogério; Grão Pará; Gravatal; Guaraciaba; Guarujá do Sul; Ibiam; Imaruí; Imbuia; Indaial; Iporã do Oeste; Itapiranga; Jaborá; Jardinópolis; Lacerdópolis; Lontras; Luzerna; Major Gercino; Major Vieira; Marema; Massaranduba; Matos Costa; Modelo; Monte Castelo; Morro Grande; Nova Itaberaba; Nova Veneza; Novo Horizonte; Ouro Verde; Paial; Paraíso; Pinhalzinho; Pinheiro Preto; Piratuba; Planalto Alegre; Ponte Alta do Norte; Presidente Nereu; Rio Rufino; Salete; Saltinho; Salto Veloso; Santa Helena; Santa Terezinha; Santiago do Sul; São Bonifácio; São João do Oeste; São José do Cerrito; Saudades; Sul Brasil; Treze de Maio; Tubarão; Tunápolis; União do Oeste; Urupema e Vargem Bonita. 
Tabela 3 - Estatísticas descritivas da eficiência por mesorregião

\begin{tabular}{llll|l|l}
\hline \multirow{2}{*}{ Mesorregião } & \multicolumn{2}{l}{ Eficiência } \\
\cline { 2 - 6 } & \multicolumn{1}{l}{ Média } & \multicolumn{1}{l}{ DP } & \multicolumn{1}{l}{ Mínimo } & \multicolumn{1}{l}{ Máximo } & \multicolumn{1}{c}{ Menor eficiência } \\
\hline \hline Serrana & 0,9913 & 0,0086 & 0,9667 & 1,0000 & Palmeira \\
Oeste Catarinense & 0,9953 & 0,0051 & 0,9809 & 1,0000 & Erval Velho \\
Vale do Itajaí & 0,9946 & 0,0048 & 0,9779 & 1,0000 & Mirim Doce \\
Grande Florianópolis & 0,9951 & 0,0031 & 0,9875 & 1,0000 & Governador Celso Ramos \\
Norte Catarinense & 0,9945 & 0,0049 & 0,9803 & 1,0000 & Irineópolis \\
Sul Catarinense & 0,9946 & 0,0053 & 0,9718 & 1,0000 & Sangão \\
Santa Catarina & 0,9945 & 0,0055 & 0,9667 & 1,0000 & Palmeira \\
\hline
\end{tabular}

Fonte: elaboração própria.

A Tabela 4 apresenta o total de municípios de cada mesorregião e quantos deles foram considerados eficientes pelo modelo DEA construído por esse trabalho. Percebe-se que $26,71 \%$ dos municípios catarinenses que compuseram a amostra foram considerados eficientes. A mesorregião Oeste Catarinense foi a que apresentou maior percentual de seus municípios na escala de eficiência, seguida posteriormente pelas mesorregiões Serrana, Vale do Itajaí, Norte Catarinense e Sul Catarinense respectivamente. E a mesorregião da Grande Florianópolis mostrou-se a com o menor percentual de seus municípios nessa escala.

Tabela 4 - Razão entre municípios eficientes e total de municípios por mesorregião

\begin{tabular}{l|c|c|c}
\multicolumn{1}{c}{ Mesorregião } & $\begin{array}{c}\text { Número total de } \\
\text { municípios }\end{array}$ & $\begin{array}{c}\text { Número de } \\
\text { municípios eficientes }\end{array}$ & $\begin{array}{c}\text { Percentual de } \\
\text { municípios eficientes }\end{array}$ \\
\hline \hline Serrana & 30 & 7 & $23,33 \%$ \\
Oeste Catarinense & 104 & 41 & $39,42 \%$ \\
Vale do Itajaí & 53 & 11 & $20,75 \%$ \\
Grande Florianópolis & 21 & 3 & $14,29 \%$ \\
Norte Catarinense & 26 & 5 & $19,23 \%$ \\
Sul Catarinense & 43 & 7 & $16,28 \%$ \\
Santa Catarina & 277 & 74 & $26,71 \%$ \\
\hline
\end{tabular}

Fonte: elaboração própria.

\subsection{Análise do impacto das práticas de governança pública}

A fim de verificar se práticas de governança pública afetam a eficiência da aplicação dos recursos públicos de educação foram selecionadas as variáveis presença de Conselhos Municipais de Educação, IRFS Social e a presença de conselhos municipais de acompanhamento e controle social do FUNDEB. O modelo de regressão Tobit foi utilizado para estabelecer quais das variáveis selecionadas são significantes para explicar a eficiência 
obtida pelo modelo construído. Foram utilizados erros padrão robustos por conta da presença de heterocedasticidade característica do modelo de regressão Tobit (GREENE, 2003; SILVA; ALMEIDA, 2012). Foi utilizado intervalo de confiança de $95 \%$ para o modelo de regressão Tobit. A Tabela 5 traz os resultados obtidos por esse modelo.

Tabela 5 - Resultado do modelo de regressão Tobit

\begin{tabular}{c|r|r|r|r|r|c}
\hline Variável & Coeficiente & \multicolumn{1}{c|}{ Z } & p-valor & Sig & VIF & Erro padrão \\
\hline \hline Constante & 0,985083 & 272,1000 & 0,0000 & $* * *$ & & 0,00361865 \\
\hline Presença de CME. & 0,00127099 & 2,0340 & 0,0420 & $* *$ & 1,017 & $6,15 \mathrm{E}-04$ \\
\hline IRFS Social & 0,0154195 & 2,4840 & 0,0130 & $* *$ & 1,015 & $6,27 \mathrm{E}-03$ \\
\hline Presença de Con. Fundeb & $3,59 \mathrm{E}-04$ & 0,4650 & 0,6419 & & 1,008 & $7,73 \mathrm{E}-04$ \\
\hline
\end{tabular}

Teste da normalidade dos resíduos-Estatística de teste: Qui-quadrado $(2)=138,582$ com p-valor $=8,0763 \mathrm{e}-032$ **Significância a $5 \%$, ***Significância a $1 \%$

Fonte: elaboração própria.

A variável presença de CME apresentou uma relação positiva com a escala de eficiência, ou seja, pode-se afirmar, ao nível de significância de 5\% (p-valor $=0,0420)$, que essa prática de governança pública impacta positivamente na eficiência da aplicação dos recursos públicos de educação. Esse resultado reflete a importância da gestão participativa dos conselhos no planejamento e na fiscalização da aplicação dos recursos municipais destinados à educação. Tal resultado corrobora o de Silva e Almeida (2012). Tal prática pode vir a ser adotada por municípios considerados ineficientes para que no futuro venham a ser eficientes quanto a aplicação dos seus recursos com educação.

Notou-se também uma relação positiva da variável IRFS Social com a escala de eficiência dos municípios catarinenses quanto à aplicação dos recursos públicos destinados à educação. Pode-se afirmar que, ao nível de significância de 5\% (p-valor =0,0130), municípios com melhores práticas de responsabilidade fiscal e social foram mais eficientes ao aplicar seus recursos com educação. Tal resultado mostra a importância de melhores práticas de gestão pública para melhoria da eficiência. Cruz, et, al. (2012), encontraram a mesma relação positiva de tal variável com o índice de transparência dos municípios.

Por fim, por mais que a presença de conselho municipal de acompanhamento e controle social do FUNDEB tenha mostrado uma relação positiva com a escala de eficiência construída por esse trabalho, essa relação não foi estatisticamente significativa, ou seja, não se pode afirmar que tal variável impacta na melhoria da eficiência dos gastos públicos com educação dos municípios catarinenses. Tal relação pode ter acontecido por conta de o FUNDEB 
representar um percentual muito pequeno do total do investimento com educação nos municípios.

Quanto ao modelo, o VIF (Fatores de Inflacionamento da Variância) mostra que nenhuma das variáveis apresentaram problemas de colinearidade. Quanto à normalidade dos resíduos, de acordo com o p-valor do teste Qui-quadrado rejeita-se a hipótese nula de normalidade, por conta do mesmo se apresentar abaixo de 5\%. Porém, conforme o teorema do limite central e levando em consideração que a amostra foi composta por mais de 100 observações no total, relaxou-se para o pressuposto da normalidade dos resíduos (GREENE, 2003).

\section{CONCLUSÃO}

Este trabalho teve como objetivo identificar se adotar práticas de governança pública incrementa a eficiência da aplicação de recursos públicos na educação nos municípios de Santa Catarina. Para tanto, foi construído um modelo utilizando a Análise Envoltória de Dados, tendo como inputs o GpcEdu\&C e a Renda, assim como outputs a TaxAt0-5 anos\%, a TaxAt5-6 anos\%, a TaxAt7-14 anos\% e a TaxAlf11-14 anos\%. Posteriormente, para a chamada "segunda fase" da DEA, selecionaram-se como variáveis proxys de práticas de governança pública: presença de CME, IRSF Social e presença de conselhos municipais de acompanhamento e controle social do FUNDEB. Utilizou-se o modelo de regressão Tobit para verificar os impactos dessas variáveis na escala de eficiência gerada pelo modelo DEA.

De acordo com os resultados do presente trabalho, pode-se afirmar que práticas de governança pública afetam a eficiência da aplicação dos recursos públicos com educação nos municípios catarinenses. A variável presença de CME apresentou uma relação positiva com a escala de eficiência dos municípios catarinenses: no nível de significância de 5\%, municípios que criam e mantêm seus conselhos municipais de Educação tendem a ser mais eficientes na aplicação dos recursos públicos nessa função do governo. Esse resultado mostra quão importantes são as práticas de governança pública participativa e como criar mecanismos que incentivem a democratização do Estado pode afetar positivamente a sociedade. Tal resultado corrobora aqueles encontrados por Silva e Almeida (2012) que encontraram relação negativa entre a presença de CME e a escala de ineficiência da aplicação dos recursos públicos com educação para os municípios do Rio Grande do Norte. 
A variável IRFS Social também apresentou uma relação positiva com a escala de eficiência dos gastos públicos de educação dos municípios catarinenses. Pode-se afirmar, no nível de significância de 5\%, que municípios com melhores práticas de responsabilidade fiscal e social conseguem maior eficiência ao aplicar seus recursos públicos com educação. Segundo constataram Cruz et al. (2012), municípios com maiores IRFS apresentam maiores índices de transparência. Por mais que tivessem objetivo diferente do presente trabalho, aqueles autores mostram que municípios com melhores práticas de gestão atingem melhores resultados.

Por mais que a variável presença de conselhos municipais de acompanhamento e controle social do FUNDEB tenha apresentado uma relação positiva com a escala de eficiência resultante do modelo DEA proposto, essa relação não foi estatisticamente significante. Isso pode ter ocorrido pelo fato de a parcela do FUNDEB representar pouca significância em relação ao total gasto com educação pelos municípios.

Vale ressaltar que os resultados dessa pesquisa podem ser relacionados somente aos municípios de Santa Catarina e não devem ser generalizados a municípios de outros estados. Contudo, os resultados obtidos com a regressão Tobit corrobora o resultado de outros autores. Portanto, atingiu-se o objetivo proposto no momento em que se identificou o impacto positivo das práticas de governança pública na escala de eficiência da aplicação dos recursos públicos de educação dos municípios catarinenses.

Assim, os resultados do presente trabalho reforçam, junto com a literatura internacional e nacional, a importância de promover boas práticas de governança pública para aumentar a eficiência da aplicação dos recursos por parte do Estado.

Espera-se que o trabalho venha a servir como inspiração para pesquisas futuras. Sugerese construir procedimentos metodológicos que façam uso de outras variáveis, modelos e orientações para a construção dessa escala aplicada para os municípios, tanto catarinenses, quanto para outros do Brasil. Também se sugere o teste de outras variáveis que representem a governança pública e a aplicação desse modelo para municípios de outros estados brasileiros e até para outros países.

\section{REFERÊNCIAS}

ABBOTT, M.; DOUCOUliAgOS, C. The efficiency of Australian universities: a data envelopment analysis. Economics of Education review, v. 22, n. 1, p. 89-97, 2003. 
AFONSO, A.; VENÂNCIO, A. The relevance of commuting zones for regional spending efficiency. Applied Economics, v. 48, n. 10, p. 865-877, 2016.

ARAÚJO JÚNIOR, J. N.; JUSTO, W. R.; ROCHA, R. M.; GOMES, S. M. F. P. O. Eficiência técnica das escolas públicas dos estados do Nordeste: uma abordagem em dois estágios. Revista Econômica do Nordeste, v. 47, n. 3, p. 61-73, 2017.

ASATRYAN, Z.; WITTE, K. Direct democracy and local government efficiency. European Journal of Political Economy, v. 39, p. 58-66, 2015.

BANKER, R. D.; CHARNES, A.; COOPER, W. W. Some models for estimating technical and scale inefficiencies in data envelopment analysis. Management Science, v. 30, n. 9, p. 1078$1092,1984$.

BELLONI, J. A. Uma Metodologia de Avaliação da Eficiência Produtiva de Universidades Federais Brasileiras. Tese (Doutorado em Engenharia de Produção) - Universidade Federal de Santa Catarina, Florianópolis, 2000.

CEPIKU, D. Public governance: research and operational implications of a literature review. Innovations in Public Management and Governance in Italy, p. 97-121, 2008

CHANG, M. Efficiency and governance of power corporations: A China and Taiwan analysis. Polish Journal of Environmental Studies, v. 23, n. 5, 2014.

CHARNES, A.; COOPER, W. W.; RHODES, E. Measuring the efficiency of decision making units. European journal of operational research, v. 2, n. 6, p. 429-444, 1978.

CNM - CONFEDERAÇÃO NACIONAL DE MUNICÍPIOS. Compreedendo e Avaliando a Gestão Municipal. Disponível em: <http://www.cnm.org.br/> . Acesso em: 15 ago. 2015

COSTA, C. C.; FERREIRA, M. A. M.; BRAGA, M. J.; ABRANTES, L. A. Fatores associados à eficiência na alocação de recursos públicos à luz do modelo de regressão quantílica. Revista de Administração Pública, v. 49, n. 5, p. 1319-1347, 2015.

CRUZ, C. F.; FERREIRA, A. C. S.; SILVA, L. M.; MACEDO, M. A S. Transparência da gestão pública municipal: um estudo a partir dos portais eletrônicos dos maiores municípios brasileiros. Revista de Administração Pública, v. 46, n. 1, p. 153-76, 2012 
CURI, M. A.; BENEDICTO, G. C.; CARVALHO, F. M.; NUINTIN, A. A.; NOGUEIRA, L. R. T. Eficiência das Universidades Federais quanto ao uso dos Recursos Renováveis. In: CONGRESSO BRASILEIRO DE CUSTOS-ABC. Anais... 2014.

DIAS, T.; CARIO, S. A. F. Governança Pública: ensaiando uma concepção. Contabilidade, Gestão e Governança, v. 17, n. 3, 2014.

FARIA, F. P.; JANNUZZI, P. M.; SILVA, S. J. Eficiência dos gastos municipais em saúde e educação: uma investigação através da análise envoltória no estado do Rio de Janeiro. Revista de administração pública, v. 42, n. 1, p. 155-177, 2008.

FARRELL, M. J. The measurement of productive efficiency. Journal of the Royal Statistical Society. Series A (General), p. 253-290, 1957.

GREENE, W. H. Econometric analysis. 5. ed. New Jersey: Prentice Hall, 2003.

HOFF, A. Second stage DEA: Comparison of approaches for modelling the DEA score. European Journal of Operational Research, v. 181, n. 1, p. 425-435, 2007.

KISSLER, L.; HEIDEMANN, F. G. Governança pública: novo modelo regulatório para as relações entre Estado, mercado e sociedade? Revista de Administração Pública, v. 40, n. 3, p. 479-499, 2006.

KNACK, S.; KEEFER, P. Institutions and economic performance: cross-country tests using alternative institutional measures. Democracy, Governance and Growth, p. 56-77, 1995.

KORMENDI, R. C.; MEGUIRE, P. G. Macroeconomic determinants of growth: cross-country evidence. Journal of Monetary economics, v. 16, n. 2, p. 141-163, 1985.

LINS, M. P. E.; MEZA, L. A. Análise envoltória de dados: e perspectivas de integração no ambiente do Apoio à Decisão. COPPE/UFRJ, 2000.

LOPES, L. S.; TOYOSHIMA, S. H. Eficiência técnica municipal na gestão dos gastos com saúde e educação em Minas Gerais: seus impactos e determinantes. Seminário sobre a economia mineira, v. 13, p. 1-24, 2008.

LYNN JUNIOR, L. E.; MALINOWSKA, A. How are Patterns of Public Governance Changing in the US and the EU? It's Complicated. Journal of Comparative Policy Analysis: Research and Practice, v. 20, n. 1, p. 36-55, 2018. 
MACEDO, M. A S.; CASA NOVA, S. P. C.; ALMEIDA, K. Mapeamento e análise bibliométrica da utilização da Análise Envoltória de Dados (DEA) em estudos em contabilidade e administração. Contabilidade, Gestão e Governança, v. 12, n. 3, 2009.

MACHADO JUNIOR, S. P.; IRFFI, G.; BENEGAS, M. B. Análise da eficiência técnica dos gastos com educação, saúde e assistência social dos municípios cearenses. Planejamento e Políticas Públicas, n. 36, 2011.

MATIAS-PEREIRA, J. A governança corporativa aplicada no setor público brasileiro. Administração Pública e Gestão Social, v. 2, n. 1, p. 109-134, 2010.

MAURO, P. Corruption and growth. The quarterly journal of economics, p. 681-712, 1995.

MELLO, J. C. C. B. S.; MEZA, L. A.; GOMES, E. G.; BIONDI NETO, L. Curso de análise de envoltória de dados. Simpósio Brasileiro de Pesquisa Operacional, v. 37, 2005.

; ___ _ _ _ _ _ _ Estudo não paramétrico da relação entre consumo de energia, renda e temperatura. IEEE Latin America Transactions, v. 6, n. 2, p. 153-161, 2008.

OLIVEIRA, A. G.; PISA, B. J. IGovP: índice de avaliação da governança pública - instrumento de planejamento do Estado e de controle social pelo cidadão. Revista de Administração Pública, v. 49, n. 5, p. 1263-1290, 2015.

OLSON JUNIOR, M.; SARNA, N.; SWAMY, A. V. Governance and growth: A simple hypothesis explaining cross-country differences in productivity growth. Public Choice, v. 102, n. 3-4, p. 341-364, 2000.

PEÑA, C. R. Um Modelo de Avaliação da Eficiência da Administração Pública através do Método Análise Envoltória de Dados (DEA). Revista de Administração Contemporânea, Curitiba, Paraná, v. 12, n. 1, p. 83-106, 2008.

REZENDE, F.; CUNHA, A.; BEVILACQUA, R. Informações de custos e qualidade do gasto público: lições da experiência internacional. Revista de Administração Pública, v. 44, n. 4, p. 959 a $992,2010$.

RHODES, R. A. W. The new governance: governing without government. Political studies, v. 44, n. 4, p. 652-667, 1996. 
RODRÍGUEZ-POSE, A; GARCILAZO, E. Quality of government and the returns of investment: Examining the impact of cohesion expenditure in European regions. Regional Studies, v. 49, n. 8, p. 1274-1290, 2015.

RONCONI, L. Governança pública: um desafio à democracia. Emancipação, v. 11, n. 1, p. 21 $34,2011$.

SILVA, A. A. P.; FERREIRA, M. A. M.; BRAGA, M. J.; ABRANTES, L. A. Eficiência na alocação de recursos públicos destinados à educação, saúde e habitação em municípios mineiros. Contabilidade, Gestão e Governança, v. 15, n. 1, p. 96-114, 2012.

SILVA, J. L. M.; ALMEIDA, J. C. L. Eficiência no gasto público com educação: uma análise dos municípios do Rio Grande do Norte. Planejamento e Políticas Públicas, n. 39, 2012.

SOUZA JÚNIOR, C. V. N. de; GASPARINI, C. E. Análise da equidade e da eficiência dos estados no contexto do federalismo fiscal brasileiro. Estudos Econômicos (São Paulo), v. 36, n. 4, p. 803-832, 2006.

SUEYOSHI, T.; GOTO, M.; OMI, Y. Corporate governance and firm performance: Evidence from Japanese manufacturing industries after the lost decade. European Journal of Operational Research, v. 203, n. 3, p. 724-736, 2010.

TEIXEIRA, L. H. G. Conselhos municipais de educação: autonomia e democratização do ensino. Cadernos de Pesquisa, v. 34, n. 123, p. 691-708, 2004.

TSCHAFFON, P.; MEZA, L. A. Assessing the efficiency of the electric energy distribution using Data Envelopment Analysis with undesirable outputs. Latin America Transactions, IEEE (Revista IEEE America Latina), v. 12, n. 6, p. 1027-1035, 2014.

WILBERT, M. D.; D’ABREU, E. C. C. F. Eficiência dos gastos públicos na educação: análise dos municípios do estado de alagoas. Advances in Scientific and Applied Accounting, v. 6, n. 3, p. 348-372, 2013 .

WHITELEY, P. F. Economic growth and social capital. Political Studies, v. 48, n. 3, p. $443-$ 466, 2000.

WILL, A. R. Eficiência dos Estados Brasileiros nos Gastos com Educação: Um Estudo Comparativo de Recursos Utilizados e Resultados Alcançados. Dissertação (Mestrado em Ciências Contábeis) - Universidade Federal de Santa Catarina, Florianópolis, 2014. 
ZHU, J. Multi-factor performance measure model with an application to Fortune 500 companies. European journal of operational research, v. 123, n. 1, p. 105-124, 2000. 